\title{
CARCINOMA EPIDERMOIDE CUTÁNEO INVASIVO A CRÁNEO. REPORTE DE UN CASO
}

\author{
Páez Adriana Min, Hinojosa Salomé2, Jaramillo Daniel2, Pareja Paola $Y^{2}$ \\ 1 Médica Neurocirujana, Servicio Neurocirugía, Hospital universitario San Jorge, \\ Pereira (Risaralda), Colombia. \\ ${ }^{2}$ Estudiante Medicina Universidad Tecnológica de Pereira, Pereira (Risaralda), Colombia. \\ Correspondencia: salomeh92@hotmail.com
}

Recibido: Septiembre 17 de 2013 Aceptado: Diciembre 9 de 2013

\begin{abstract}
Resumen
Dentro del grupo de cáncer cutáneo no melanoma (CCNM), el Carcinoma cutáneo de células escamosas (CE) representa el 25\% siendo el segundo más frecuente, con una incidencia a lo largo de la vida del 7 y $11 \%$. El Carcinoma Epidermoide (CE) es más agresivo que el Carcinoma basocelular (CBC). También pueden invadir estructuras profundas, causando significativa destrucción local. Presentamos el caso clínico de una mujer de 71 años que había sido previamente intervenida por diagnóstico anatomopatológico de carcinoma epidermoide de cuero cabelludo moderadamente diferenciado. Posteriormente presentó amplia recidiva local en zona frontal con invasión de diploe craneal y espacio epidural. Fue intervenida quirúrgicamente y el defecto secundario se reparó con colgajo e injertos. La biopsia confirmó bordes libres. Sin embargo un mes después, la paciente presentó invasión del cáncer a techo de la órbita. Se describe el tratamiento quirúrgico realizado por los servicios de Cirugía Plástica y Neurocirugía. Se reporta este caso clínico, ya que la incidencia de cáncer en la piel ha aumentado en los últimos años, por las mayores áreas expuestas al sol y mayor longevidad. Sin embargo su evolución es detenida la mayoría de las veces con un diagnóstico y tratamiento oportuno, aspectos con los que no contó la paciente, terminando en una excepcional invasión intracraneal, implicación poco frecuente en la práctica clínica.
\end{abstract}

Palabras clave: Carcinoma de células escamosas, cirugía plástica, cuero cabelludo, hueso frontal, hueso parietal.

\section{INVASIVE SQUAMOUS CELL CARCINOMA TO SKULL. A CLINICAL CASE PRESENTATION}

\begin{abstract}
Within non-melanoma skin cancer (NMSC) group, squamous cell carcinoma (SCC) represents 25\%, with an incidence over life of 7 to $11 \%$. Epidermoid Carcinnoma is more aggressive than basal cell carcinoma (BCC). It also can invade deep structures, causing significant local destruction. The case of a 71 years old woman, who was previously pathologically diagnosed with moderately differentiated epidermoid carcinoma in the scalp, is reported. There is a high rate of frontal recurrence with invasion to skull and epidural space. Surgery was performed and the secondary defect was repaired with flap and graft. Biopsy showed free edges. However after one month, the patient presented cancer invasion roof of the orbit. The surgical treatment by plastic surgery and
\end{abstract}


neurosurgery is described. The incidence of skin cancer has increased in recent years, because of larger sun-exposed areas and increased longevity. However, its evolution is slowed down most of the times with early diagnosis and appropriate treatment, aspects to which the patient had not had access to, ending in exceptional intracranial invasion, rare implication in clinical practice.

Keywords: Squamous Cell Carcinoma, plastic surgery, scalp, frontal bone, parietal bone.

\title{
CARCINOMA EPIDERMÓIDE CUTÂNEO A CRÂNIO: RELATO DE CASO CLÍNICO
}

\begin{abstract}
Resumo
No grupo de câncer de pele não melanoma, o carcinoma espinocelular cutâneo (CEC) é responsável por $25 \%$ dos casos e o segundo mais comum, com uma incidência de 7 a $11 \%$. O CEC é mais agressivo do que o carcinoma basocelular (CBC), também podem invadir estruturas mais profundas, causando destruição local significativa. Relata-se o caso de uma mulher de 71 anos, intervinda cirurgicamente pelo diagnóstico patológico de moderamente diferenciado de carcinoma de células escamosas no couro cabeludo. Apresentou extensa recorrência local na frente posteriormente com invasão da cabeça e o espaço epidural. O defeito secundário foi feito com retalho e enxerto, tomou-se biopsia, confirmaram-se bordes livres pela patologia, porém um mês depois, a paciente presentou invasão do câncer para o teto da orbita. Faz se relato da cirurgia feita por Cirurgia Plástica e Neurocirurgia. A incidência de câncer de pele tem aumentado nos últimos anos, pelas maiores áreas expostas da pele, porém, sua evolução é detida com a avaliação e tratamento atual. O caso clinico apresenta um paciente com diagnóstico e tratamento tardio, com invasão na cabeça excepcional, situação pouco freqüente na pratica clínica.
\end{abstract}

Palavras-chave: Carcinoma de células escamosas, cirurgia plástica, couro cabeludo, osso frontal, osso parietal.

\section{Introducción}

El Carcinoma cutáneo de células escamosas (SCC) es un tumor maligno de queratinocitos en la capa espinosa de epidermis. Está clasificado dentro del grupo de cáncer cutáneo de tipo no melanoma, al igual que el carcinoma basocelular siendo este último más frecuente, seguido por el SCC (1) que representa el 25\%, con una incidencia a lo largo de la vida del 7 y $11 \%$ (2). Se ha reportado que 72 a 100,8 personas por cada 100.000 habitantes, presen$\tan$ SCC (3). Ocurre principalmente en personas ancianas (60-70 años) (4) y en el género masculino (5). El SCC de cabeza y cuello (CECC) es la quinta neoplasia más común en el mundo. (6)

Dentro de los factores de riesgo para desarrollar SCC, se encuentran la exposición a rayos UV, la infección por el virus del papiloma humano (VPH), tabaquismo y zonas con inflamación crónica. (5)
Existen diferentes factores de riesgo relacionados con la recurrencia del tumor y metástasis, entre ellos el tamaño, la profundidad, invasión, el tipo histológico, diferenciación, inmunosupresión, infección por VPH, zonas de alto riesgo y expresión de genes tumorales. (7)

Para el tratamiento de SCC, se recomienda radioterapia (RT), terapia superficial, y cirugía, siendo esta última principalmente utilizada en SCC de alto riesgo, en donde la cirugía de Mohs tiene mayor eficacia. Sin embargo por su alto costo, se utiliza la resección quirúrgica seguida de evaluación patológica postoperatoria de los márgenes (POMA) (8), con un margen lateral mínimo de $6 \mathrm{~mm}$ (1), Como tratamiento adyuvante la RT es el tratamiento estándar. (9)

Para el manejo de los defectos secundarios generados tras la extirpación tumoral en cuero cabelludo, se utilizan colgajos locales, expansión y reordenación de tejido. (8) Sin 
embargo se prefieren colgajos libres para zonas extensas, como los colgajos de omentum. (10) Otras publicaciones han usado técnicas microquirúrgicas o colgajos locales pediculados sin mucha repercusión funcional ni estética, siendo esta última más sencilla. (11)

\section{Caso clínico:}

Presentamos el caso de una paciente de 71 años con antecedentes de actividad laboral al aire libre desde la infancia, sin uso de prácticas protectoras contra rayos solares (no uso de protección solar, ni camisa de manga larga o sombrero e incluso cabello corto), la cual acude a consulta por recidiva de un SCC en cuero cabelludo.

La paciente fue intervenida hace ocho meses por lesión de $1 \mathrm{~cm}$ de diámetro en región frontoparietal derecha, de bordes irregulares, levemente pruriginosa con diagnóstico de "SCC moderadamente diferenciado", con resección quirúrgica total.

Siete meses después es valorada en tercer nivel de atención y presenta lesión de 10 x $7 \mathrm{~cm}$, ulcerada en región frontotemporoparietal derecha, sin adenopatías palpables ni alteración neurológica. Se encuentra en RM una lesión infiltrante de piel, cuero cabelludo, tabla ósea y compromiso epidural. (figura 1).

En valoración conjunta entre los servicios de neurocirugía y cirugía plástica, se decide llevar a cirugía para resec- ción del tumor por craneotomía supratentorial y cubrimiento del tejido expuesto mediante colgajo e injertos. En el procedimiento se encontró lesión supratentorial con compromiso epidural y severo compromiso de hueso con infiltración a lo largo de todo el borde a nivel temporal derecha, frontal basal, fronto-orbitario y techo de la órbita. Se realiza resección del cuero cabelludo afectado, acompañada de trepanación hemi-frontoparietal y exposición de la duramadre.

Macroscópicamente no se evidencia invasión a duramadre. (Figura 2) La biopsia intraoperatoria realizada, informó 5 semanas más tarde bordes libres de lesión. El defecto secundario producido tras la resección tumoral fue cubierto con colgajo fasciocutáneo, axial pediculado de la arteria temporal, de rotación y avance más injertos libres de piel abdominal. (Figura 3)

En cuanto al progreso de colgajo, presentó sufrimiento cutáneo del extremo más distal, que evolucionó de forma favorable con tratamiento conservador.

Pasado 1 mes de la intervención. Se realiza TAC de control sin que se haya registrado signos de recidiva tumoral.

Consulta al mes siguiente, y pese a que el servicio de patología reportó bordes libres de lesión, la paciente manifiesta disminución en agudeza visual en ojo derecho, además de evidente edema en zona frontotemporal derecha, lo que generó sospechas de recidiva tumoral severa, diagnostico

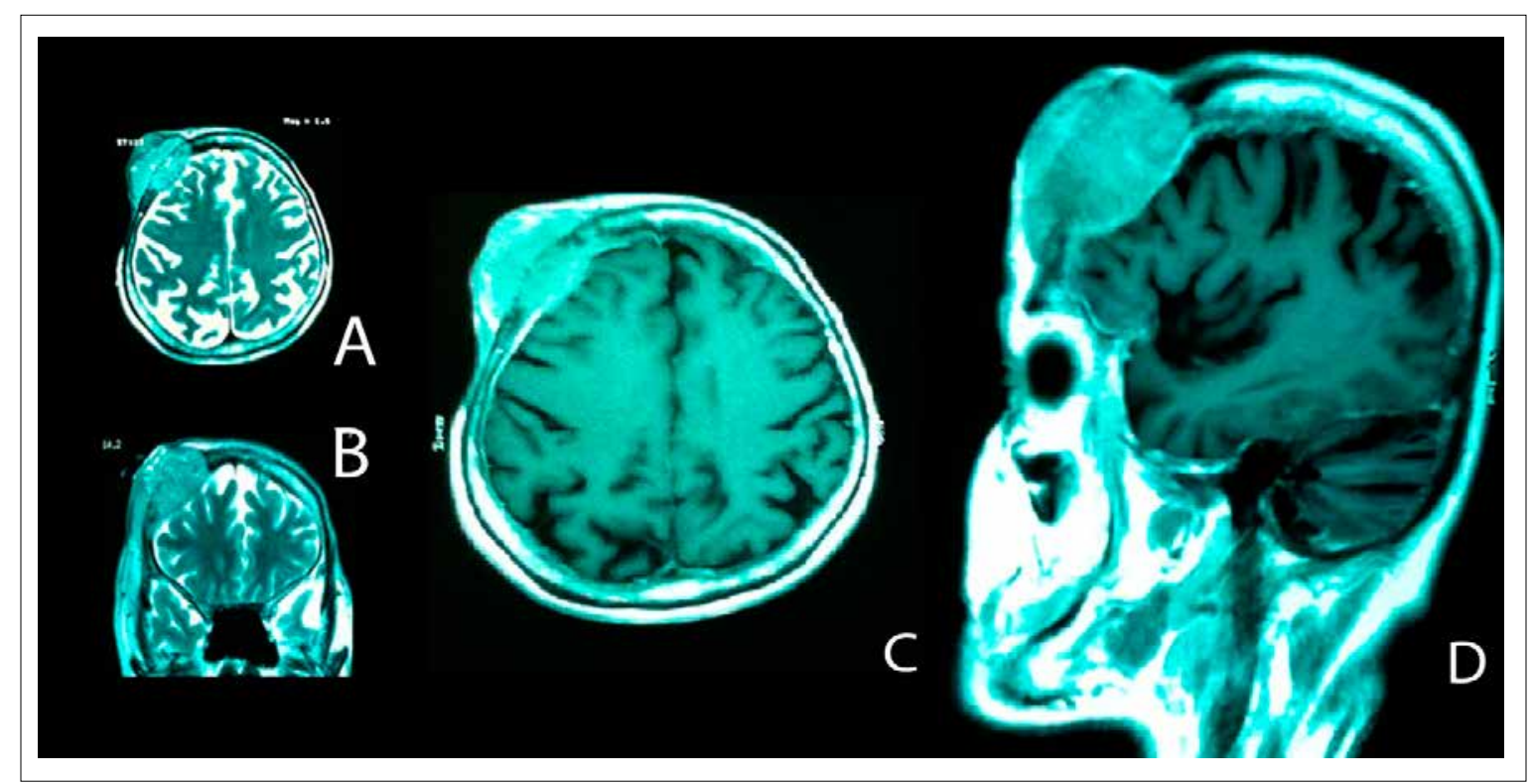

Figura 1. A IRM Axial T2 con lesión frontal derecha, con infiltración ósea y epidural. B IRM Coronal T2 con lesión con compromiso de cuero cabelludo, diploe y epidural frontal derecha. C. IRM Corte Axial T2 con contraste que presenta lesión frontal derecha, con infiltración ósea y epidural. D.IRMCorte parasagital derecho T1 con contraste. 


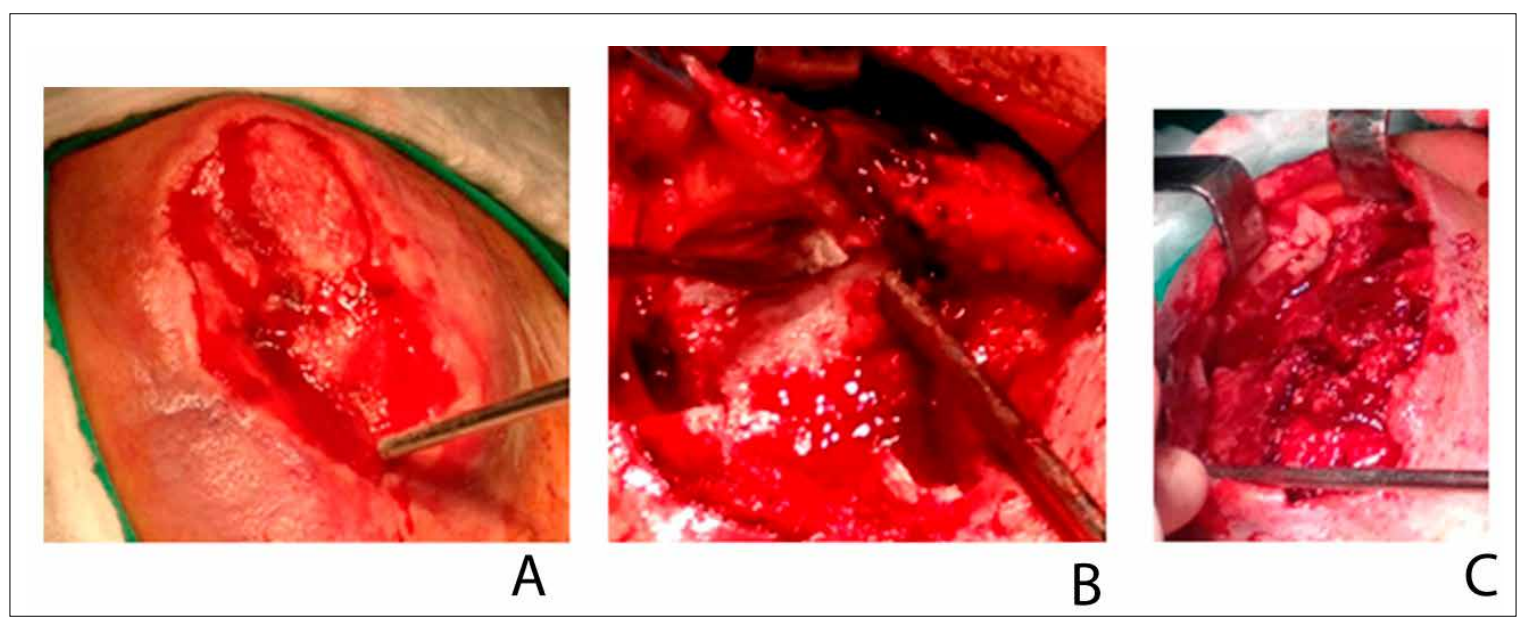

Figura 2. A Lesión Frontal derecha con compromiso de piel, cuero cabelludo y masa solida friable e infiltrante B Lesión con compromiso de hueso frontal (Superior) y compromiso de espacio epidural (Inferior) $C$ Lesión invasiva frontal derecha y con severo compromiso epidural frontal.

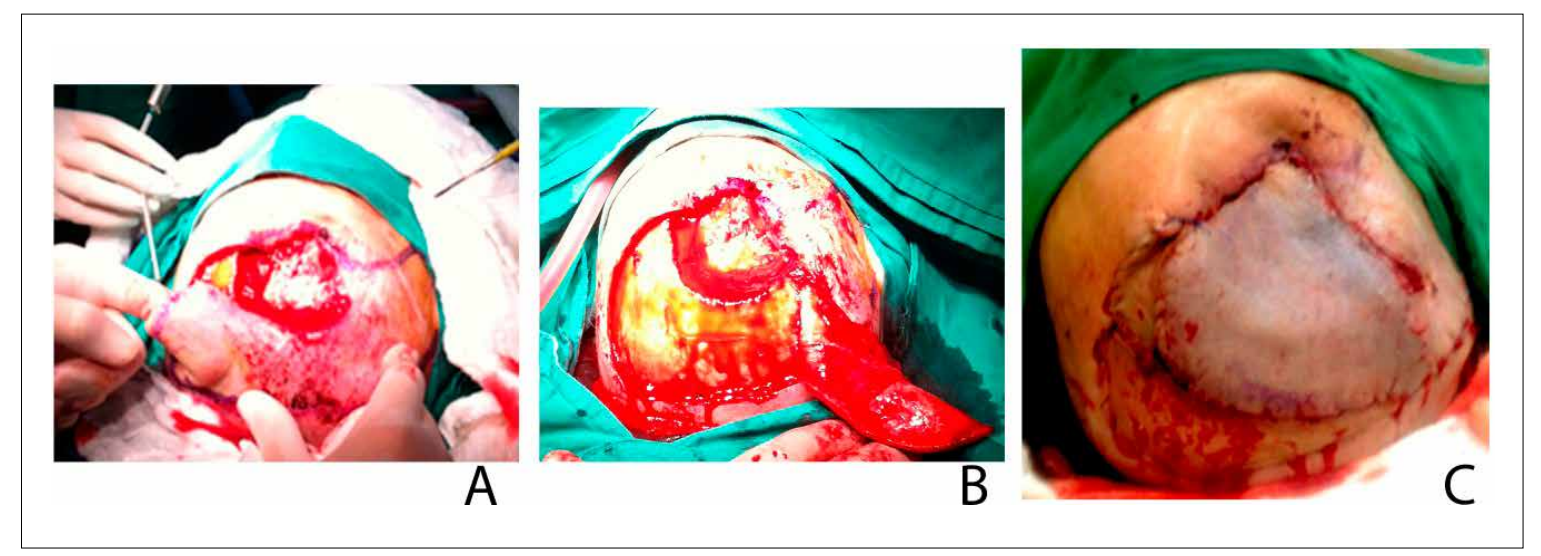

Figura 3. A, B Colgajo fasciocutáneo, axial pediculado de la arteria temporal, de rotación y avance. C Resultado Colgajo fasciocutáneo más injertos libres.

que se confirmó por RM posteriormente indicando que la lesión invade techo y pared lateral de orbita derecha con compromiso de fosa pteriogopalatina y extensión frontal medial y temporoparietal.

Dada la evolución se remite a cuarto nivel para cirugía radical y reconstructiva.

\section{Discusión}

Dentro del grupo de cáncer cutáneo no melanoma (CCNM), el más frecuente es el carcinoma basocelular, seguido del SCC, el cual es un tumor maligno de queratinocitos en la capa espinosa de epidermis (1) que representa el 25\% de los CCNM, con una tasa de incidencia de
$72 / 100.000$ para las mujeres y $100,8 / 100.000$ para los hombres (3). Se reporta una incidencia a lo largo de la vida del 7 y $11 \%(2)$

La paciente reportó trabajo en el campo desde la infancia, sin uso de prácticas protectoras contra rayos solares. Un estudio realizado en Colombia, reveló que el 59\% de los pacientes con SCC, declaró haber tenido alguna ocupación laboral al aire libre antes de los 15 años de edad, donde las actividades relacionadas con el campo (agricultura y ganadería) ocuparon el primer lugar dentro de los pacientes. El mismo estudió demostró que después de los 30 años de edad, el uso de prácticas protectoras, como el sombrero, camisa de manga larga y el protector solar fue bajo, con porcentajes de 24\%, 23\%, 1.29\% respectivamente (13). Estableciendo así, como el trabajo en la 
agricultura y el no uso de prácticas protectoras pudo haber sido un factor desencadenante en la paciente.

Una revisión durante 5 años de 3493 casos de cáncer cutáneos malignos, encontró que la cara (54\%) y el cuero cabelludo $(13,9 \%)$ eran la localización más frecuente, en donde el SCC en cuero cabelludo tenía una prevalencia del $8,3 \%$ y de estos, el $25,9 \%$ eran mujeres (5).

En cuanto a la distribución por género, se sabe que el sexo masculino, es el más afectado, principalmente en el pabellón auricular y el cuero cabelludo, debido a que el pelo masculino tiene menos efecto protector, a diferencia de las mujeres en donde las zonas más afectadas son el pecho y los miembros inferiores, ya que esta zona es comúnmente expuesta (13). Dado lo anterior podemos analizar que la paciente reportó haber llevado cabello corto a lo largo de su vida, lo que podría explicar la ubicación de su patología.

Respecto a la edad, el SCC se encuentra más frecuentemente a los 74,4 $\pm 12,4$ años (5). Según un estudio en un hospital colombiano, el promedio de edad fue de 71 años (12), por lo tanto se puede observar que la paciente, se encuentra dentro del marco de edad más afectado.

En relación a la diferenciación, la paciente presento un SCC moderadamente diferenciado. Una revisión entre 1997-2009 de pacientes con SCC en cuero cabelludo, encontró que el $42 \%$ de los tumores se clasificaban como bien diferenciados, 31\% moderadamente diferenciados, $16 \%$ poco diferenciados (14).

En el presente reporte de caso, se encontró que la paciente tuvo 2 recidivas tumorales. Según la literatura, se ha definido como un SCC con riesgo de recurrencia, metástasis de ganglios linfáticos y/o metástasis a distancia cuando tiene un riesgo mayor del $5 \%$, determinado sobre la base de las características del tumor y factores del paciente. (15) Para lo anterior la National Comprehensive Cancer Network (NCCN), proporciona una lista consenso de factores para determinar el riesgo de recurrencia del SCC (tabla 1), de los cuales la paciente presenta seis factores de alto riesgo de recurrencia, lo que explicarían las repetidas recidivas (8).

La literatura confirma la importancia del tamaño del tumor, en donde $2 \mathrm{~cm}$ es el límite a partir donde se tiene mayor tendencia a metástasis, e incluso cuando son mayores a $2 \mathrm{~cm}$, la probabilidad de recurrencia se duplica y la probabilidad de metástasis se triplica (7).

Un estudio realizado en 2008, encontró que el 16\% presentaba metástasis cuando el tumor tenía una profundidad mayor de $6 \mathrm{~mm}$ de profundidad (16). Incluso en la séptima edición de la clasificación TNM para SCC de la American Joint Committee on Cancer (AJCC), incluye el grosor del tumor y el nivel de invasión en profundidad, para la cual la paciente se encuentra en estadio III, T3NOM0. (tablas 2, 3) (17)

Tabla 1. Factores de alto riesgo de recurrencia en Carcinoma cutáneo de células escamosas, según la National Comprehensive Cancer Network (NCCN).

\begin{tabular}{|c|c|c|}
\hline & Alto riesgo & Bajo riesgo \\
\hline \multirow{3}{*}{ Tamaño / Ubicación } & ÁREA M: $20 \mathrm{~mm}$ & ÁREA M: $<20 \mathrm{~mm}$ \\
\hline & ÁREA L: $10 \mathrm{~mm}$ & ÁREA L: $<10 \mathrm{~mm}$ \\
\hline & ÁREA H: $6 \mathrm{~mm} 3$ & ÁREA $\mathrm{H}:<6 \mathrm{~mm} 3$ \\
\hline Borde & Pobremente definido & Bien definido \\
\hline Primario vs Recurrente & Recurrente & Primario \\
\hline Inmunosupresión & + & - \\
\hline Sitio de RT previa o de proceso inflamatorio crónico & + & - \\
\hline Rápido crecimiento del tumor & + & - \\
\hline Síntomas neurológicos & + & - \\
\hline Grado o diferenciación & Moderadamente o pobremente diferenciado & Bien diferenciado \\
\hline Subtipo adenoides (acantolítica), adenoescamoso o desmoplásico. & ++ & - \\
\hline Profundidad & Nivel de Clark IV, V o $2 \mathrm{~mm}$ & Nivel Clark I, II, III o <2mm \\
\hline La invasión perineural o vascular & + & - \\
\hline \multicolumn{3}{|c|}{$\begin{array}{l}\text { ÁREA M: Area de la Cara (centro de la cara, parpados, cejas, periorbital, nariz, labios (cutáneo y vermellón) barbilla, mandíbula, piel/surcos preau- } \\
\text { riculares y postauriculares, oreja) }\end{array}$} \\
\hline \multicolumn{3}{|l|}{ ÁREA L: Mejillas, frente, cuero cabelludo y cuello } \\
\hline \multicolumn{3}{|l|}{ ÁREA H: Tronco y extremidades } \\
\hline
\end{tabular}


Tabla 2. Clasificación TNM según la American Joint Committee on Cancer (AJCC), séptima edición.

\begin{tabular}{|c|c|}
\hline T: Tamaño del tumor & $\begin{array}{l}\text { Tx El tumor primario no se puede localizar } \\
\text { T0 No existe evidencia del tumor primario } \\
\text { Tis Carcinoma in situ } \\
\text { T1 Carcinoma de menos de } 2 \mathrm{~cm} \text { en su diámetro mayor de extensión con menos de dos factores } \\
\text { de riesgo* } \\
\text { T2 Carcinoma de más de } 2 \mathrm{~cm} \text { en su diámetro mayor o tumor de cualquier tama no con más de dos } \\
\text { factores de riesgoa } \\
\text { T3 Invasión tumoral del maxilar, mandíbula, órbita o hueso temporal } \\
\text { T4 Invasión tumoral ósea o extensión perineural en la base craneal }\end{array}$ \\
\hline N: afectación de ganglios linfáticos & $\begin{array}{l}\text { Nx Los ganglios linfáticos regionales no pueden ser valorados } \\
\text { N0 No existen metástasis en los ganglios linfáticos regionales } \\
\text { N1 Metástasis en un único ganglio linfático ipsilateral al tumor de } 3 \mathrm{~cm} \text { o menos en su mayor dimensión } \\
\text { N2 Metástasis en un ganglio linfático ipsilateral al tumor, mayor de } 3 \mathrm{~cm} \text { en su diámetro mayor pero } \\
\text { menor de } 6 \mathrm{~cm} \text {. o múltiples ganglios ipsilaterales que no excedan los } 6 \mathrm{~cm} \text { o múltiples ganglios } \\
\text { bilaterales o contralaterales no mayores de } 6 \mathrm{~cm} \\
\text { N2a Metástasis en un ganglio linfático ipsilateral al tumor, mayor de } 3 \mathrm{~cm} \text { en su diámetro mayor, pero } \\
\text { menor de } 6 \mathrm{~cm} \\
\text { N2b Múltiples ganglios ipsilaterales que no excedan los } 6 \mathrm{~cm} \\
\text { N2c Múltiples ganglios bilaterales o contralaterales no mayores de } 6 \mathrm{~cm} \\
\text { N3 Metástasis en un ganglio linfático mayor de } 6 \mathrm{~cm} \text { en su diámetro mayor }\end{array}$ \\
\hline M: metástasis a distancia & $\begin{array}{l}\text { M0 No metástasis a distancia } \\
\text { M1 Metástasis a distancia }\end{array}$ \\
\hline \multicolumn{2}{|c|}{$\begin{array}{l}\text { Se excluye SCC de párpados } \\
\text { * Factores de alto riesgo para tumor primario: }\end{array}$} \\
\hline \multicolumn{2}{|c|}{$\begin{array}{l}\text { Profundidad/invasión > 2mm de grosor. Nivel de Clark >IV; Invasión perineural; Localización anatómica: Pabellón auricular o labios; Diferenciación } \\
\text { (pobremente diferenciado o indiferenciado). }\end{array}$} \\
\hline
\end{tabular}

Tabla 3.Estadiaje del carcinoma epidermoide cutáneo, según la American Joint Committee on Cancer (AJCC), séptima edición.

\begin{tabular}{|c|c|c|c|}
\hline Estadío & T & N & M \\
\hline 0 & Tis & N0 & M0 \\
\hline I & T1 & N0 & M0 \\
\hline II & T2 & N0 & M0 \\
\hline \multirow{2}{*}{ III } & T3 & N0 & M0 \\
& T1 - T3 & N1 & M0 \\
\hline \multirow{3}{*}{ IV } & T1 - T3 & N2 & M0 \\
& Cualquier T & N3 & M0 \\
& Cualquier T & Cualquier N. & Cualquier N. \\
& \multicolumn{2}{|c}{ M1 } \\
\hline
\end{tabular}

En cuando al grado de diferenciación, los CEC se pueden dividir en grado I cuando el tumor tiene más del $75 \%$ de sus células diferenciadas, grado II entre 50 y $75 \%$ del tumor diferenciado, grado III (pobremente diferenciado) cuando las células diferenciadas corresponden al 25 y $50 \%$ y grado IV cuando son menos del $25 \%$, lo que en la práctica clínica se le llama bien, moderadamente o pobremente diferenciado (18).
Igualmente se ha relacionado la infección por VPH tipo beta con el SCC. Se calcula el 90\% de los pacientes inmunosuprimidos están infectados por este tipo $(19,20)$; se sabe que la inmunosupresión relativa asociada a la edad en pacientes ancianos se ha relacionado con la infección por un mayor número de serotipos de VPH (20), aspecto que no se puede ignorar en la paciente dada su edad

La literatura por muchos años ha clasificado como zonas de alto riesgo aquellas ricamente inervadas y vascularizadas, con poco tejido subcutáneo, que podrían facilitar la invasión de estructuras profundas y la rápida diseminación, donde algunos han incluido el cuero cabelludo (5).

En cuanto a la patología metastásica se sabe que el SCC en cuero cabelludo ocupa el quinto lugar en frecuencia en afección parótida lo cuales empeoraría el pronóstico (21) al relacionarse un peor control de la afección y donde las adenopatías metastásicas cervicales empeoran el pronóstico (22).

En tratamiento de los SCC de alto riesgo, existe un modelo proporcionado por la (NCCN) (8), donde la terapia de elección es la cirugía de Mohs, una técnica quirúrgica 
que incluye durante la cirugía un control histológico de los bordes por un patólogo (23) pero debido a sus altos costos y a que no es útil en SCC con invasión ósea (24), en la paciente, se utilizó la técnica POMA (resección quirúrgica convencional seguida de evaluación patológica postoperatoria de los márgenes). Las indicaciones para POMA son: SCC de alto riesgo, re-intervencón en BCC y SCC bajo riesgo en zona $\mathrm{L}$, si hay márgenes positivos tras POMA (8). Dicha técnica se hace resecando la lesión en bloque con un margen lateral mínimo de $6 \mathrm{~mm}$ (1), con lo cual se consigue una tasa de erradicación de 95\% (25); en el presente caso la resección se basó en la observación macroscópica de friabilidad y a partir de allí se calculó el margen.

En cuando al manejo con RT, la NCCN, recomienda que se reserve para candidatos no quirúrgicos, pacientes en edad avanzada, tumores en zona de alto riesgo de hasta $15 \mathrm{~mm}$ de diámetro y tumores en lugares de riesgo intermedio de hasta $20 \mathrm{~mm}$ de diámetro (8).

El valor de la radiación postoperatoria en la reducción de la tasa de recurrencia en pacientes de alto riesgo ha sido ampliamente aceptado. Se recomienda en casos de afectación perineural, cuando los márgenes de tejido son positivos después de la cirugía, lográndose así controles locales cercanos al 100\%. Sin embargo, un 30\% de los pacientes de alto riesgo sufren recurrencia regional y un $25 \%$ metástasis a distancia. Se estima que un $70-80 \%$ de los pacientes presentan recurrencia dentro de los 2 primeros años (8). Un estudio de la Universidad de Florida encontró un $72 \%$ de supervivencia a los 5 años de la enfermedad en los pacientes tratados con cirugía y RT postoperatoria en comparación con el 59\% tratados solamente con cirugía o RT (26).

En otras modalidades de tratamiento, se encuentra el 5-Fluorouracilo (5-FU) para el tratamiento de la queratosis actínica, pero no es el Gold Standar para el manejo del cáncer (27) La terapia fotodinámica está recomendada para el tratamiento de queratosis actínica y Terapia Fotodinámica para la enfermedad de Bowen (27), o en pacientes en edad avanzada con grandes lesiones multifocales o recurrentes en quienes la resección quirúrgica esté contraindicada $(28,29)$. El manejo con electrodesecación debido a sus tasas de recurrencia en tumores de alto riesgo, se reserva para los tumores pequeños superficiales, bien definidos y que vayan solo hasta la dermis (30).

En los defectos secundarios generados tras la extirpación tumoral se prefiere colgajos libres, mediante utilización de colgajos de omentum (10). Sin embargo, existen diversas opciones como reordenación de tejido local el cual se utiliza para defectos de hasta 50\% del cráneo (8) y los defec- tos resultantes se pueden cerrar con injertos de piel. Los expansores de tejido son una opción válida para tumores pequeños ya que cuando los defectos son muy grandes, se puede retrasar el tratamiento. Sin embargo, se presentan tasas de complicaciones de hasta el 25\% (8). También se han usado técnicas microquirúrgicas que ofrecen mejores opciones reconstructivas, con mayor morbilidad; por eso se han implementado técnicas con colgajos locales pediculados (11), sin demasiada repercusión funcional ni estética, siendo esta última más sencilla, aspecto que se tuvo en cuenta en el manejo del caso.

Defectos óseos pueden ser reconstruidos ya sea con un autoinjerto o aloinjerto, Split de calota, injertos óseos y cubrir pequeñas y medianos defectos (31), al igual que cerámicas y polímeros tales como metacrilato de metilo para cubrir y restaurar el contorno del calvario cuando los defectos son grandes o cuando el material autólogo no está disponible (32). En la paciente se decidió esperar el reporte de patología con bordes libres para proceder con la reconstrucción craneal.

En cuanto a la recidiva de la enfermedad, un estudio realizado entre 1997-2009 en la Universidad de Calgary en Canadá, reportó tasas de recurrencia pese al tratamiento de 3,8\% (14) Otro trabajo realizado en Inglaterra demostró una tasa de recurrencia local del $6 \%$ y una tasa de metástasis de un 7\%, y todos los pacientes que presentaron recurrencia de la enfermedad, lo hicieron dentro de los 18 meses de la cirugía primaria (33). Lastimosamente, la paciente se encontró dentro del pequeño porcentaje de individuos que presenta recurrencia.

\section{Conclusiones}

-La intervención evitó la infiltración del proceso tumoral a cerebro y un deterioro irreversible en la paciente.

- La correcta y rápida evaluación de la biopsia es indispensable para el tratamiento, ya que anticipa a la evidencia clínica e imagenológica.

- El sistema de seguridad social en Colombia genera obstáculos para un rápido diagnóstico y tratamiento.

\section{Agradecimientos}

Damos el más sincero agradecimiento al Hospital Universitario San Jorge por permitirnos el acceso a historia clínica, así como también al servicio de Neurocirugía por el trato y calidad humana realizado con la paciente. 


\section{Referencias}

1. Gonzalo N., Vial G., Ibarra A., Cabezas L. Tumores de cabeza y cuello. Rev Med Clin Condes. 2007; 18(4): 408-18

2. de Vries E1, van de Poll-Franse LV, Louwman WJ, de Gruijl FR, Coebergh JW. Predictions of skin cancer incidence in the Netherlands up to 2015.Brit J Dermatol. 2005; 152:481

3. Aceituno P, Buendia A, Arias S, Serrano S. Evolución de la incidencia del cáncer de piel en el período 1978-2002. Actas Dermosifiliogr. 2010;101:39-46.

4. Petter G, Haustein UF. Squamous cell carcinoma of the skin - histopathological features and their significance for the clinical outcome.J EurAcadDermatolVenereol. 1998 Jul;11(1):37-44

5. Andrade P. Epidemiology of basal cell carcinomas and squamous cell carcinomas in a Department of Dermatology - a 5 year review. An Bras Dermatol. 2012;87(2):212-19

6. Parkin DM, Bray F, Ferlay J, Pisani P. Estimating the world cancer burden: Globocan 2000. Int J Cancer 2001;94:153-6

7. Nuño A., Vicente F, Pinedo F. López J. Carcinoma epidermoide cutáneo de alto riesgo..Actas Dermosifiliogr. 2012;103(7):567$-578$

8. Miller SJ. Basal cell and squamous cell skin cancers. Clinical Practice Guideline in oncology. NatlComprCancNetw. 2010 Aug;8(8):836-64

9. Bernier J, Cooper JS, Pajak TF. Defining risk levels in locally advanced head and neck cancers: a comparative analysis of concurrent postoperative radiation plus chemotherapy trials of the EORTC and RTOG). Head Neck 2005;27:843-50

10. Navarro F. Reconstrucción de cuero cabelludo con colgajo libre de Omentum. Rev Esp Cirug Oral y Maxilofac. 2004; 26: 249-256.

11. Taboada A, Prieto A, Couto I, Brea B, González E.Carcinoma basocelular invasivo de cuero cabelludo. Caso clinic. Neurocirugia.2010; 21(5) :396-400.

12. Nova JA, Sanchez G, Peña EB. Características epidemiológicas de pacientes con carcinoma escamocelular cutáneo en el Centro Dermatológico Federico Lleras Acosta, Bogotá, Colombia.Rev Asoc Colomb Dermatol. 2011;19: 212-217

13. Rigel DS. Cutaneous ultraviolet exposure and its relationship to the development of skin cancer. J Am AcadDermatol. 2008;58(5 Suppl 2): S129-32.

14. Elzinga K. Morbidity of invasive squamous cell carcinoma of the scalp. J Am Coll Surg. 2012; 215, 3: S83-S84

15. Veness MJ, Palme CE, Morgan GJ. High-risk cutaneous squamous cell carcinoma of the head and neck: results from 266 treated patients with metastatic lymph node disease. Cancer. 2006;106:2389---96.

16. Brantsch KD, Meisner C, Schönfisch B, Trilling B, Wehner-Caroli $J$, Röcken $\mathrm{M}$, et al. Analysis of risk factors determining prognosis of cutaneous squamous-cell carcinoma: a prospective study. Lancet Oncol. 2008;9:713---20.

17. Farasat S, Yu SS, Neel VA, Nehal KS, Lardaro T, Mihm MC, et al. A new American Joint Committee on Cancer staging system for cutaneous squamous cell carcinoma: Creation and rationale for inclusion of tumor (T) characteristics. J Am Acad Dermatol. 2011;64:1051---9.

18. Cassarino DS, Derienzo DP, Barr RJ. Cutaneous squamous cell carcinoma: a comprehensive clinicopathologic classification. Part one. J Cutan Pathol. 2006;33:191206.

19. de Koning MN, Weissenborn SJ, Abeni D, BouwesBavinck JN Euvrard S, Green AC, et al. Prevalence and associated factors of betapapillomavirus infections in individuals without cutaneous squamous cell carcinoma. J Gen Virol. 2009;90: 1611---21.

20. Patel AS, Karagas MR, Pawlita M, Waterboer T, Nelson HH. Cutaneous human papillomavirus infection, the EVER2 gene and incidence of squamous cell carcinoma: a case-control study. Int $J$ Cancer. 2008;122: 2377---9.

21. Bumpous J. Metastatic cutaneous squamous cell carcinoma to the parotid and cervical lymph nodes: treatment and outcomes. Curr Opin Otolaryngol Head Neck Surg. 2009;17: 122---5.

22. O'Brien CJ, McNeil EB, McMahon JD, Pathak I, Lauer CS, Jackson MA. Significance of clinical stage, extent of surgery, and pathologic findings in metastatic cutaneous squamous carcinoma of the parotid gland. Head Neck. 2002;24: 417---22.

23. Alonso T. Cirugía de Mohs: nuestros primeros cien pacientes. Actas Dermosifiliogr. 2008; 99 : 275-280

24. Ross AS, Whalen FM, Elenitsas R, Xu X, Troxel AB, Schmults $\mathrm{CD}$. Diameter of involved nerves predicts outcomes in cutaneous squamous cell carcinoma with perineural invasion: an investigator-blinded retrospective cohort study. Dermatol Surg. 2009;35:1859---66.

25. Brodland DG, Zitelli JA. "Surgical margins for excision of primary cutaneous squamous cell carcinoma," J Am AcadDermatol. 1992;27(2 Pt 1):241-8.

26. delCharco J.O et al "Carcinoma of the skin metastatic to the parotid area lymph nodes," Head \& Neck. 1998; 20, no. 5, pp. 369-373,

27. Gurudutt V.V, Genden E.M. Cutaneous Squamous Cell Carcinoma of the Head and Neck. Journal of Skin Cancer. 2011

28. . V. G. Schweitzer. Photofrin-mediated photodynamic therapy for treatment of aggressive head and neck nonmelanomatous skin tumors in elderly patients. Laryngoscope. 2001; 111 (6): 1091-1098.

29. Marmur E, Schmults C, Goldberg D. A review of laser and photodynamic therapy for the treatment of nonmelanoma skin cancer. Dermatologic Surgery. 2004; 30(2): 264-271.

30. Tull S, Nunley K, Sengelmann R. Nonsurgical treatment modalities for primary cutaneous malignancies. Dermatologic Surgery. 2008;34(7): 859-872.

31. Rieger KE, Linos E, Egbert BM, Swetter SM. Recurrence rates associated with incompletely excised low-risk nonmelanoma skin cancer. J Cutan Pathol 2010;37:59-67.

32. Silverman MK, Kopf AW, Grin CM. Recurrence rates of treated basal cell carcinomas. Part 1: Overview. J Dermatol Surg Oncol. 1991; 17:713-718.

33. Jenkins G.W. Anatomical restrictions in the surgical excision of scalp squamous cell carcinomas: does this affect local recurrence and regional nodal metastases?.British Journal of Oral and Maxillofacial Surgery. 2012; 50: S16. 